\title{
EstAcqua: Proposta de solução integrada de Hardware, Software e Internet das Coisas para monitoramento ambiental
}

\author{
Alan Afif Helal ${ }^{1}$, Roberto Colistete Júnior ${ }^{2}$, \\ Fábio da Cunha Garcia ${ }^{3}$, Gilberto Fonseca Barroso ${ }^{3}$, Rodolfo da Silva Villaça ${ }^{1}$ \\ ${ }^{1}$ Programa de Pós-Graduação em Informática (PPGI) \\ Universidade Federal do Espírito Santo (UFES) - Vitória - ES - Brasil \\ ${ }^{2}$ Departamento de Química e Física \\ Universidade Federal do Espírito Santo (UFES) - Alegre - ES - Brasil \\ ${ }^{3}$ Departamento de Oceanografia e Ecologia \\ Universidade Federal do Espírito Santo (UFES) - Vitória - ES - Brasil \\ alan@helal.com.br, roberto.colistete@ufes.br, garciafc2007@gmail.com \\ gilberto.barrosodufes.br, rodolfo.villaca@ufes.br
}

\begin{abstract}
With the expansion of the Internet of Things, several solutions for monitoring are available in the market. However, most solutions use proprietary, costly software and do not offer online monitoring, which difficults access to data and prevents to take actions in a preventive way. This article presents EstAcqua, an integrated solution of low cost hardware and software that uses concepts of Internet of Things with LoRaWan, which main objective is to monitor environmental and oceanographic data from surface and submerged sensors, featuring remote access in real time and low energy consumption. Tests were performed in real environments to show the feasibility of the solution.
\end{abstract}

Resumo. Com a expansão da Internet das Coisas, diversas soluções para monitoramento estão disponíveis no mercado. Entretanto, a maioria das soluções utilizam software proprietário, de custo elevado e não oferecem monitoramento online, dificultando o acesso aos dados e impedindo que ações sejam tomadas de forma preventiva. Este artigo apresenta a EstAcqua, uma solução integrada de hardware e software de baixo custo que utiliza conceitos de Internet das Coisas com LoRaWan, cujo objetivo principal é monitorar dados ambientais e oceanográficos de sensores de superfície e submersos, podendo ser acessada remotamente em tempo real e tendo baixo consumo de energia. Foram efetuados testes em ambientes reais para mostrar que a solução é viável.

\section{Introdução}

Segundo o relatório da National Aeronautics and Space Administration (NASA) ${ }^{1}$ a temperatura global aumentou $1.0^{\circ} \mathrm{C}$ desde o ano de 1880 , sendo que os últimos 18 anos mais quentes ocorreram depois do ano 2000. Além do aumento da temperatura, tem-se também o aumento do nível dos mares, a diminuição das camadas polares e o aumento da emissão de dióxido de carbono. Diante disso, monitorar o meio ambiente é de extrema importância.

\footnotetext{
${ }^{1}$ https://climate.nasa.gov
} 
Dentre os ecossistemas aquáticos os lagos têm sido considerados como efetivos sentinelas de mudanças ambientais ao integrar em seus sistemas físicos, químicos e biológicos respostas da variabilidade dos fluxos de energia e matéria, inclusive possibilitando o registro de longo prazo de mudanças climáticas [Adrian et al. 2009, Schindler 2009]. A necessidade de avaliação das tendências dos processos de estratificação e desestratificação térmica, hidrodinâmica, distribuição de nutrientes e gases dissolvidos, como oxigênio, além de produtividade primária e secundária demandam o monitoramento contínuo e de longo prazo de variáveis chave destes processos. A partir da análise de dados e a geração de informações subsidia-se sistemas de suporte à decisão (SSD) para gestão ambiental lacustre quanto aos problemas de eutrofização, assoreamento, contaminação e a consequente perda de bens e serviços ambientais proporcionados pelos ecossistemas aquáticos [Straskraba and Tundisi 2000]. Nessa linha, o Programa Global Lake Ecological Observatory Network - GLEON tem como foco o desenvolvimento de projetos científicos inovadores para coleta, compartilhamento e interpretação de dados em alta resolução de sensores para compreensão, predição e comunicação do papel e resposta dos lagos em um ambiente global em mudança.

Atualmente, profissionais e pesquisadores fazem monitoramento de áreas urbanas e rurais a partir da obtenção de variáveis ambientais (e.g. climáticas, limnológicas, oceanográficas), visando a criação de um banco de dados com a evolução das condições ambientais. Para isso utilizam-se estações meteorológicas e através das quais se obtém dados climáticos como temperatura do ar, precipitação da chuva, velocidade do vento, umidade e iluminância, assim como sensores hidrológicos para a obtenção de variáveis como temperatura da água, concentração de oxigênio dissolvido, $\mathrm{pH}$, turbidez, entre outras.

Entretanto, os equipamentos mais comercializados para a medição dessas variáveis apresentam um grande problema: são equipamentos proprietários, de elevado custo, com código fonte fechado e sem interoperabilidade com equipamentos de outros fabricantes. Dessa forma, o usuário fica restrito à aquisição de produtos do mesmo fabricante que já utiliza. Ou seja, caso outro fabricante lance um produto de melhor qualidade, com mais funcionalidades ou de menor custo, ele não será compatível com os outros produtos já adquiridos.

Dentre os procedimentos mais utilizados, os sensores são instalados no local de medição e fazem a coleta dos dados através de um datalog e, após um período, normalmente de um mês, deve-se voltar até o sensor para coletar os dados registrados. Nesse ínterim, caso o sensor seja furtado e/ou danificado, perde-se todos os dados coletados. Além disso, os sistemas de monitoramento em tempo contínuo convencionais não dispõe deavisos prévios no caso de uma variável ultrapassar um valor limite, como por exemplo, se a concentração de oxigênio dissolvido for inferior a 2,0 mg/L, implica em estresse fisiológico para os peixes. Caso o local de instalação dos equipamentos seja de difícil acesso, a tarefa de instalar o equipamento e coletar os dados se torna ainda mais exaustiva.

Estamos vivendo uma época de proliferação de objetos inteligentes com capacidade de sensoriamento, processamento e comunicação [Loureiro 2016]. Nos últimos anos ocorreu uma miniaturização e redução dos preços dos sensores tornando mais fácil a tarefa de monitorar. Com a facilidade de obtenção de sensores de baixo custo e con- 
sumo, diversas soluções de monitoramento utilizando conceitos de Internet das Coisas (do inglês Internet of Things (IoT)) estão sendo desenvolvidas. Atualmente temos 11 bilhões de dispositivos IoT e, segundo a Forbes ${ }^{2}$, o ano de 2018 será de grandes avanços nessa área.

Nesse contexto, este artigo apresenta a EstAcqua, uma estação com sensores para monitoramento ambiental e oceanográfico, atualmente de superfície que coletam dados de pressão atmosférica, umidade, temperatura e iluminância, além de submersos para coletarem temperaturas em diferentes profundidades. Todos os sensores estão conectados a um microcontrolador LoPy $4^{3}$ e o envio dos dados é realizado usando LoRaWan ${ }^{4}$.

Essa estação, além de possuir uma arquitetura aberta de implementação, com uma solução integrada de software e hardware de baixo custo, tem como diferenciais: (i) possibilidade de instalação em locais remotos e sem acesso à Internet ou sinal de celular; (ii) longo alcance de transmissão (podendo chegar a poucos quilômetros); (iii) acesso aos dados pela Internet; (iv) criação de gráficos mostrando a variação dos dados, com possibilidade de criação de alarmes para notificar situações que necessitam de atenção, tornando possível tomar ações preventivas; (v) fácil instalação e utilização; e (vi) excelente autonomia de bateria e ilimitada com adição de painel solar.

Para validação do protótipo da estação ambiental, foram realizadas medições na lagoa situada dentro da Universidade Federal do Espírito Santo (UFES) e transmitidos os dados para o Departamento de Oceanografia e Ecologia, situado a 130 metros do local das medições. Também foram efetuados testes no Lago Terra Alta, no município de Linhares, no norte do estado do Espírito Santo, onde o protótipo foi colocado em uma balsa instalada no meio do lago e os dados coletados foram transmitidos para a margem do lago situada a até $3,2 \mathrm{~km}$ de distância, como mostra a Figura 1.

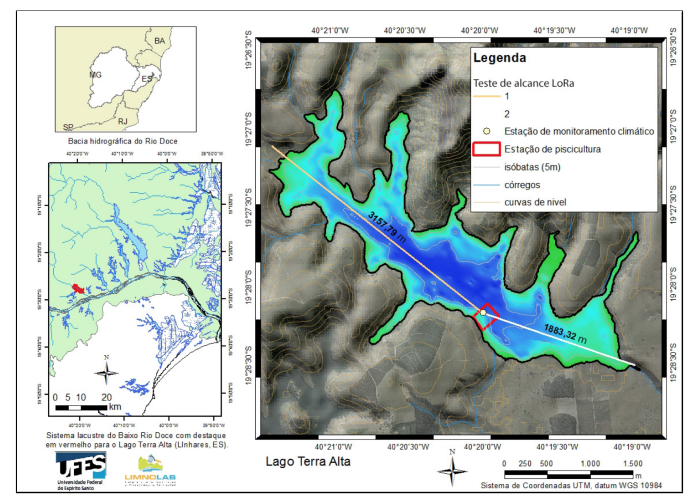

Figura 1. Localização da EstAcqua no lago Terra Alta (Linhares, ES) e experimento de alcance de dados via LoRaWan

O restante deste artigo está dividido da seguinte forma: Na Seção 2, serão apresentados e discutidos trabalhos relacionados. Na Seção 3, será apresentada a arquitetura, as características e os desafios encontrados na implementação da EstAcqua. Na Seção 4,

\footnotetext{
${ }^{2}$ https://www.forbes.com/sites/bernardmarr/2018/01/04/the-Internet-of-things-iot-will-be-massive-in2018-here-are-the-4-predictions-from-ibm

${ }^{3}$ https://pycom.io/hardware/lopy4-specs

${ }^{4}$ Protocolo que define a arquitetura da tecnologia LoRa (https://www.lora-alliance.org)
} 
será detalhada a avaliação da EstAcqua. A Seção 5 conclui o artigo e faz indicações de trabalhos futuros.

\section{Trabalhos Relacionados}

A popularização de microcontroladores de fácil programação e interface amigável proporcionou a criação de diversas soluções de hardware integrada com software, dentre elas para a área de monitoramento ambiental. Com a facilidade de integração com os microcontroladores removeu-se a barreira que outrora existia: a dificuldade eletrônica para que seja possível integrar o microcontrolador com outros dispositivos.

No trabalho de Thobias [Tose 2012] foi desenvolvido um protótipo para monitoramento de estações de esgoto com Arduino conectado a sensores de temperatura, umidade e gases, utilizando uma tecnologia de comunicação sem fio, ZigBee, para transmissão dos dados. Entretanto, essa é uma abordagem de alto custo financeiro, com baixo alcance (devido à frequência de operação), muito sensível a interferências de outros dispositivos, com baixa autonomia de bateria e dependente de um computador localizado próximo ao local de instalação do protótipo. A EstAcqua se diferencia por ter um alcance de transmissão significativamente maior, funcionar com bateria e a possibilidade de funcionamento sem a necessidade de um computador próximo.

Já o trabalho de Fabio [de Oliveira 2017] mostra a criação de um magnetômetro utilizando conceitos de Internet das Coisas com uma interface web para visualização dos dados online. O magnetômetro conta com sensores de temperatura para ajudar nas medidas realizadas e armazena em um banco de dados em um cartão micro SD. O presente trabalho se diferencia por usar LoRaWan para transmissão dos dados e ser focado em monitoramento ambiental e oceanográfico.

George Mois [Mois et al. 2017] fez um estudo comparativo de três soluções diferentes de sensores inteligentes para o monitoramento de dados ambientais utilizando transmissão sem fio WiFi e Bluetooth. Por se tratar de tecnologias de baixo alcance, o emprego dessas soluções é voltado para ambientes fechados, como escritórios. O diferencial em relação a esse trabalho é a possibilidade de uso interno ou externo com transmissão de longo alcance.

$\mathrm{Na}$ grande maioria dos trabalhos encontrados na literatura, as soluções são para curto alcance, dependentes de um computador próximo para se obter os dados dos sensores ou de uma fonte de energia externa para energizar o equipamento. Um exemplo é o trabalho proposto por Bharathkumar [Bharathkumar et al. 2017], em que se utiliza um microcontrolador PIC P $^{5}$ conectado a um sensor de temperatura e umidade com a possibilidade de envio de mensagens de texto para um celular. A solução proposta neste trabalho utiliza um hardware mais robusto, com mais sensores e software aberto, o que possibilita notificações através de $e$-mail, por exemplo, de acordo com a necessidade do usuário.

Dessa forma, um diferencial da arquitetura proposta neste artigo é a sua aderência ao modelo de Internet das Coisas, onde os dados dos sensores podem ser acessados através de uma interface $w e b$ ou de um aparelho celular, sem a necessidade de infraestrutura existente no local de instalação da EstAcqua. Além disso, caso haja alguma indisponibilidade

\footnotetext{
${ }^{5}$ https://www.microchip.com/design-centers/microcontrollers
} 
de acesso à Internet, os dados coletados são salvos em cartão micro SD, evitando a perda de dados.

\section{Arquitetura de Hardware e Software}

A arquitetura da EstAcqua pode ser dividida em quatro partes: um ou mais nós transmissores de dados, receptor de dados, nuvem de Internet das Coisas (IoT Cloud) e interação com usuário. A Figura 2 ilustra essas quatro partes.

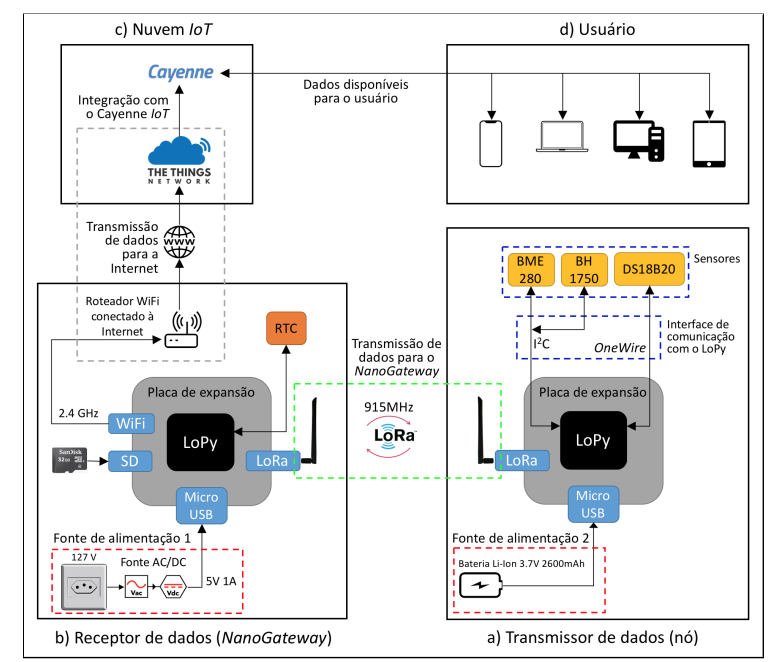

Figura 2. Arquitetura da EstAcqua

\subsection{Hardware}

A arquitetura de hardware é composta principalmente pelos blocos Transmissor de dados e Receptor de dados representados na Figura 2:

- Receptor de dados (NanoGateway): responsável por receber os pacotes enviados pelos transmissores de dados. É alimentado por uma fonte de alimentação com saída DC de 5V e $1 \mathrm{~A}$ e está conectado à Internet para encaminhar os dados recebidos para um servidor online.

- Transmissor de dados (nó): responsável pela coleta e processamento dos dados obtidos dos sensores. Possui sensores de superfície de pressão, umidade, temperatura e iluminância e sensores submersos de temperatura. É alimentado por uma bateria de $2.600 \mathrm{mAh}$ com saída de 3,7 V.

Tanto o NanoGateway como cada nó utilizam um microcontrolador LoPy4 (ou LoPy) pois já possui integrado WiFi, LoRa(Wan) e Bluetooth. Além disso o LoPy4 possui um modo de economia de energia, deep sleep, em que um co-processador ULP (Ultra Low Power) é utilizado para monitorar as portas de entrada/saída do LoPy4, os canais do conversor analógico-digital e controlar a maioria dos periféricos internos com um consumo baixíssimo de energia. Adicionalmente é utilizada uma placa de expansão que possui entradas USB, cartão de memória e conector para a bateria, facilitando a interface com o LoPy4.

O nó é responsável pela coleta e processamento os dados dos sensores conectados a ele e pela transmissão via LoRaWan para o NanoGateway. Essa unidade está conectada a sensores ambientais e oceanográficos, sendo eles: (i) sensor BME280 para coletar 
pressão atmosférica, umidade e temperatura; (ii) sensor BH1750 para medir iluminância; (iii) sensores de temperatura DS18B20 para coletarem a temperatura da água ao longo de diferentes profundidades.

Os sensores BME280 e BH1750 utilizam interface de comunicação $I^{2} \mathrm{C}$ e os sensores oceanográficos submersos, DS18B20, utilizam interface de comunicação OneWire. O protótipo do nó construído possui um BME280, um BH1750 e diversos DS18B20 para medir a temperatura em diferentes profundidades entre subsuperfície até $15 \mathrm{~m}$.

O NanoGateway recebe os pacotes transmitidos pelos nós (transmissores de dados) e envia para um IoT Cloud para que os dados fiquem acessíveis de forma online. Como o NanoGateway fica ativo o tempo todo, ele deve ser ligado à energia elétrica em um local próximo a uma rede sem fio com acesso à Internet. Ainda conta com um DS3231 via $I^{2} \mathrm{C}$, um Real Time Clock (RTC) com bateria externa, regulado por cristal de quartzo e com compensação de ano bissexto, para que o sistema mantenha seu horário sempre sincronizado mesmo em caso de interrupções na alimentação. O IoT Cloud escolhido foi a The Things Network (TTN) ${ }^{6}$, por se tratar de uma comunidade colaborativa com mais de 35 mil membros e quase 20 mil aplicações desenvolvidas, além de facilitar a integração dos dados recebidos com bancos de dados, HTTP e Cayenne.

Para diminuir as chances de perda de dados, o NanoGateway possui um cartão micro SD de 32GB de armazenamento onde são escritos todos os dados recebidos.

Na Figura 3 são mostradas: o nó coletando a temperatura em diferentes profundidades de uma lagoa, bem como a temperatura, umidade e pressão do ambiente da lagoa; o NanoGateway; o hardware do interior do nó; e microcontroladores LoPy(4) utilizados.

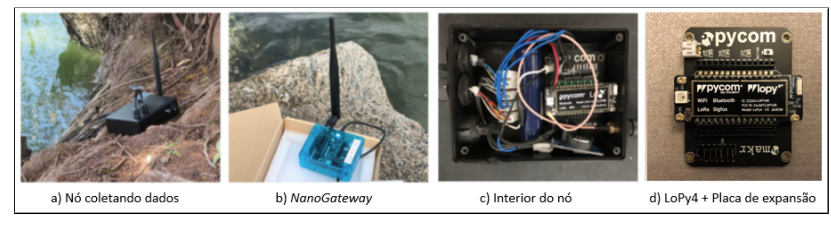

Figura 3. Hardware da EstAcqua

\subsection{Software}

Com relação ao software embarcado na EstAcqua, trata-se de dois códigos-fonte $\mathrm{Mi}$ croPython ${ }^{7}$ distintos, sendo um específico para o NanoGateway e outro para o nó, ambos criados pelos autores. O nó possui um código adaptativo que reconhece automaticamente quando algum sensor é conectado ou desconectado. Como cada nó foi projetado para ser instalado em um local de difícil acesso, sendo alimentado por uma bateria, foi necessário otimizar o código-fonte para que se tenha o menor consumo de energia.

Após a instalação e inicialização do nó, ele entra no modo ativo, que dura aproximadamente 2.9 segundos, onde (i) realiza as medições; (ii) compacta os dados para a criação de um pacote no formato Cayenne $L P P^{8}$; (iii) envia o pacote para o NanoGateway; (iv) grava os dados coletados no cartão micro SD; (v) e entra em modo deep sleep, onde permanece por 10 minutos. Após esse tempo ele acorda novamente e repete o processo.

\footnotetext{
${ }^{6}$ https://www.thethingsnetwork.org

${ }^{7}$ https://www.micropython.org

${ }^{8}$ https://www.thethingsnetwork.org/docs/devices/arduino/api/cayennelpp.html
} 
Já o NanoGateway possui um código para receber pacotes apenas dos nós que se autenticam através de Authentication By Personalisation (ABP). Após receber um pacote, os dados recebidos são gravados em um cartão micro SD e enviados para a TTN para visualização dos dados de forma online, tanto via web ou através do celular usando o aplicativo Cayenne conforme ilustrado na Figura 2.

\section{Avaliação e Resultados}

Para avaliar o protótipo e sua viabilidade foram realizados os seguintes testes: (i) comunicação via LoRa(Wan), para verificar como o protótipo se comporta em diferentes situações e decidir quais parâmetros utilizar na comunicação; (ii) interface de monitoramento com a nuvem, a fim de analisar como os dados enviados pelo nó são enviados para o IoT Cloud e são disponibilizados para o usuário; (iii) avaliação no lago Terra Alta, visando analisar o comportamento do protótipo em locais de difícil acesso e sem comunicação com a Internet; (iv) autonomia da bateria, objetivando calcular um valor teórico do tempo sem a necessidade de recorrer a uma fonte externa de energia para recarregá-la e; (iv) avaliação dos sensores utilizados, comparando-os com sensores comerciais.

\subsection{Comunicação via LoRa(Wan)}

Para comunicação via LoRa(Wan) é necessário selecionar alguns parâmetros:

- Frequência de transmissão: frequência utilizada para a comunicação entre os dispositivos. No Brasil é utilizada a banda de $915 \mathrm{MHz}(902-907,5 \mathrm{MHz}, 915-928$ $\mathrm{MHz}$ ), escolhemos 903,9 MHz;

- Fator de espalhamento (SF): LoRa opera com SF de 7 a 12, sendo 7 o que proporciona maior taxa de transmissão e menor alcance, e 12 a menor taxa de transmissão e maior alcance;

- Largura de banda (BW): LoRa utiliza três BW: $125 \mathrm{kHz}, 250 \mathrm{kHz}$ (não permitido no Brasil) e $500 \mathrm{kHz}$, sendo $125 \mathrm{kHz}$ com menor taxa de transmissão e maior alcance e $500 \mathrm{kHz}$ com maior taxa de transmissão e menor alcance;

- Code Ratio (CR): proporção dos dados enviados que não são redundantes. LoRa utiliza 4/5 (maior taxa de transmissão e menor alcance), 4/6, 4/7 ou 4/8 (menor taxa de transmissão e maior alcance).

O tamanho do payload do pacote transmitido via LoRa(Wan) depende da combinação dos parâmetros acima. Dessa forma é feito uma solução de compromisso entre distância máxima, velocidade de transmissão, taxa de perda e tamanho do pacote.

Para decidir quais parâmetros utilizar foram realizados testes na Praia de Camburi, no município de Vitória, no Estado do Espírito Santo, onde foi posicionado o nó em um píer e o NanoGateway em outro píer situado a $2.8 \mathrm{~km}$ de distância para simular os efeitos de transmissão a nível do mar, por se tratar de um cenário similar ao encontrado na lagoa de Terra Alta. Foram selecionadas 28 combinações de SF, BW e CR partindo da configuração de menor alcance e alta taxa de transmissão $(\mathrm{SF}=7, \mathrm{BW}=500 \mathrm{kHz}$ e $\mathrm{CR}=$ 4/5) para a de maior alcance e baixa taxa de transmissão ( $\mathrm{SF}=12, \mathrm{BW}=125 \mathrm{kHz}$ e CR =4/8). Para cada configuração foram enviados 100 pacotes para fazer uma estatística de perda de pacotes. Foi então selecionada a configuração $\mathrm{SF}=8, \mathrm{BW}=125 \mathrm{kHz}$ e $\mathrm{CR}=$ $4 / 5$, pois apresentou uma taxa de perda menor que $1 \%$ e um payload máximo de 51 bytes - representando uma taxa de perda baixíssima para uma longa distância e comportando os dados de todos os sensores em um único pacote. 


\subsection{Interface de Monitoramento via Nuvem}

Depois foram realizados testes em campo na lagoa situada dentro da Universidade Federal do Espírito Santo (UFES), monitorando a temperatura da lagoa em diferentes profundidades, pressão, umidade, temperatura e iluminância do ambiente. Os dados foram enviados para o NanoGateway localizado no Departamento de Oceanografia, a 130m distância da lagoa e $11 \mathrm{~m}$ de altura, estando conectado à rede sem fio da UFES. O nó foi posicionado próximo à Lagoa e bastou ligá-lo para que os dados fossem transmitidos para o NanoGateway, ratificando assim a sua fácil instalação e uso.

Os dados transmitidos pelo nó no teste da UFES foram visualizados através do site do Cayenne e com o aplicativo Cayenne para dispositivos móveis. A Figura 4 mostra a forma com que os dados são disponibilizados de forma online para o usuário. Também é possível a criação de gráficos com os valores lidos pelos sensores para acompanhar a evolução dos valores e a criação de alarmes para enviar $e$-mails caso algum dos sensores esteja com leitura acima ou abaixo do valor configurado pelo usuário. A Figura 5 mostra os gráficos de temperatura externa e submersa na lagoa da UFES, onde pode-se perceber a inércia de variação da temperatura da água da lagoa em relação à temperatura ambiente ${ }^{9}$.

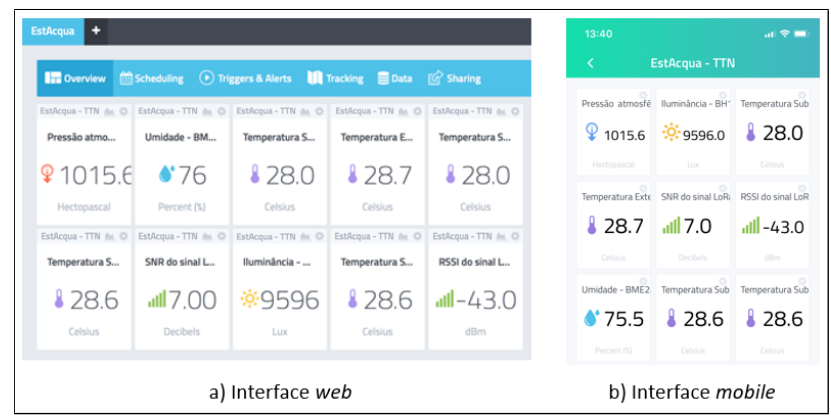

Figura 4. Interface web e mobile do Cayenne com os dados acessados online

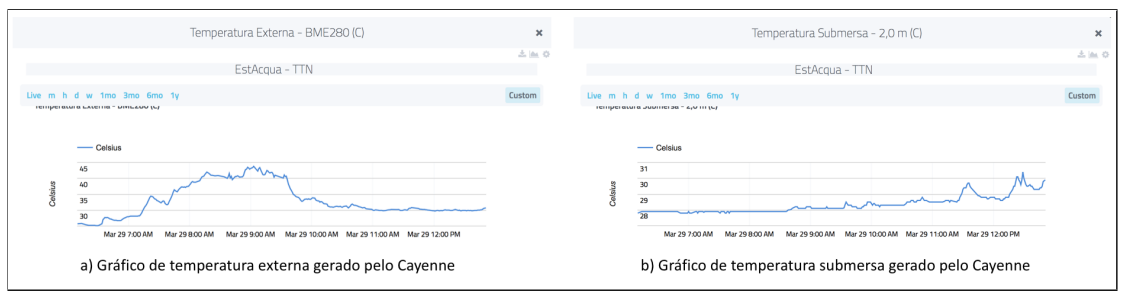

Figura 5. Gráficos de temperatura gerados pelo Cayenne

\subsection{Avaliação no Lago Terra Alta}

Outro teste foi realizado no Lago Terra Alta (área de $3,9 \mathrm{~km}^{2}$ e profundidade máxima de $22,1 \mathrm{~m}$ ) por se tratar de um importante ecossistema lacustre profundo sob estresse ambiental da atividade de piscicultura [Barroso et al. 2012] com produção estimada em 2017 de 550 toneladas de tilápia. Desde junho de 2014, o lago vem sendo monitorado com variáveis climáticas a cada 15 minutos e temperatura da água em oito profundidades a

\footnotetext{
${ }^{9}$ Link do Cayenne de acesso público para visualização dos dados coletados pela EstAcqua: https://cayenne.mydevices.com/shared/5ac1490bd8b6585016379d30
} 
cada 60 minutos. O objetivo é avaliar o efeito dos fatores climáticos sobre o regime térmico do lago e propriedades hidroquímicas e hidrobiológicas. O lago Terra alta fica em uma localidade remota e sem sinal de celular ou conexão com a Internet. Na psicultura existe uma balsa onde sensores ambientais já estão instalados. No lago Terra Alta, o monitoramento de variáveis limnológicas produz dados contínuos da estrutura térmica da coluna de água, conforme Figura 6. Essas informações são importantes para o estudo da influência de mudanças climáticas na estrutura térmica da coluna de água e seus reflexos para a ecologia desses ecossistemas. O protótipo da EstAcqua foi instalado nessa balsa e foram realizados testes movimentando o NanoGateway pela lagoa, chegando até sua margem localizada a $3,15 \mathrm{~km}$ de distância.

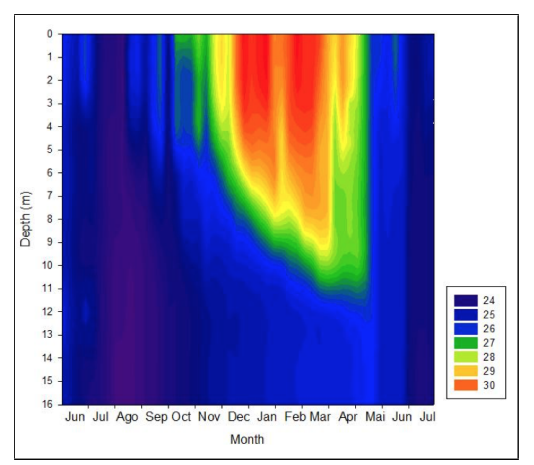

Figura 6. Diagrama profundidade/tempo para a variação da temperatura na coluna de água do lago Terra Alta

O teste mostrou que em local remoto a instalação do protótipo foi rápida e fácil e mesmo sem comunicação com a internet não houve perda de dados pois os mesmos foram gravados no cartão micro SD, confirmando sua viabilidade mesmo em ambientes sem conexão com a internet ou sinal de celular.

A Figura 7 mostra a balsa instalada no lago Terra Alta e o nó instalado na balsa no lago Terra Alta.

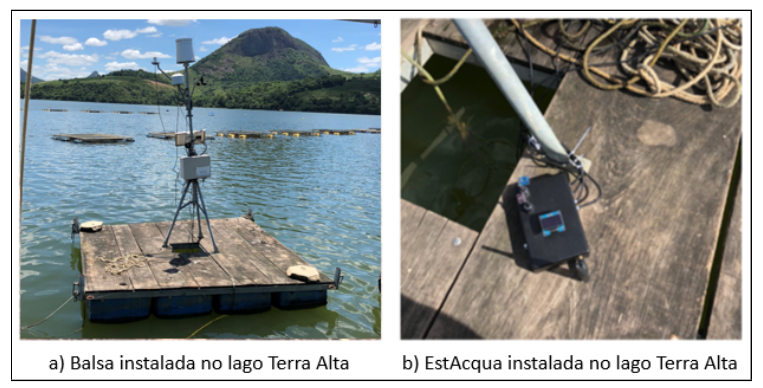

Figura 7. Balsa localizada no lago Terra alta e o nó na balsa coletando dados

\subsection{Autonomia de Bateria}

Para estimar a autonomia da bateria $T_{b}$ através de cálculos teóricos são necessárias a capacidade da bateria $C$ (em mAh), a corrente elétrica consumida no modo ativo $I_{a}$ (em $\mathrm{mA}$ ), no modo inativo (deep sleep) $I_{d}(\mathrm{em} \mathrm{mA}$ ) e a fração de tempo $D(\mathrm{em} \%$ ) em que o 
sistema está no modo ativo (denominada duty cycle ou ciclo de trabalho) :

$$
T_{b}=\frac{100 C}{24\left[I_{a} D+I_{d}(100-D)\right]},
$$

que mostra que se deve minimizar $I_{a}$ e $I_{d}$ para maximizar a autonomia da bateria $T_{b}$.

Foi utilizado o sensor de corrente contínua INA219 para medir a corrente consumida no modos ativo e multímetro no modo inativo, resultando em consumos médios $I_{a}=(40,0 \pm 1,3) \mathrm{mA}$ e $I_{d}=(0,43 \pm 0,04) \mathrm{mA}$, respectivamente. Utilizando o tempo em que o nó EstAcqua permanece no estado ativo, 2, 9s, tempo de acordar do modo inativo, carregar os módulos, configurar LoRAWan, ler sensores e enviar os dados via LoRaWan, em relação ao período do ciclo de operação de 600 s, obtemos um ciclo de trabalho $D \simeq 0,483 \%$. Empregando uma bateria com capacidade nominal de $2.600 \mathrm{mAh}$ resulta em longa autonomia de bateria $T_{b} \simeq(171 \pm 12)$ dias, i.e., entre 159 e 183 dias. Se adicionamos janela temporal de downlink (recebimento de dados do servidor) LoRaWan, são mais 2,6 s totalizando 5,5 s no estado ativo, $D \simeq 0,917 \%$ e autonomia de bateria mais curta, $T_{b} \simeq(134 \pm 8)$ dias, entre 126 e 142 dias. Vide a Figura 8 que mostra claramente o comportamento não-linear da Equação (1) de autonomia da bateria.

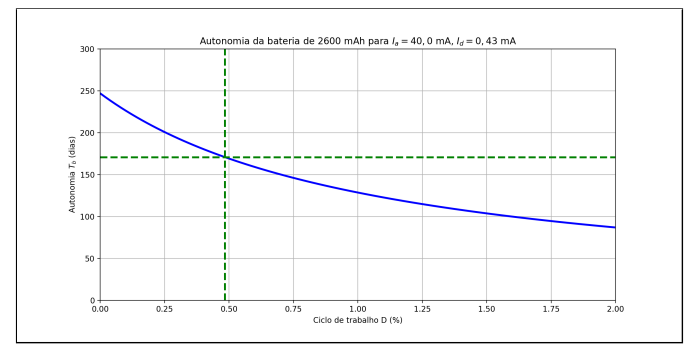

Figura 8. Autonomia da bateria de 2.600 mAh versus ciclo de trabalho para correntes de modo ativo $I_{a}=40,0 \mathbf{m A}$ e de deep sleep $I_{d}=0,43 \mathbf{m A}$.

\subsection{Avaliação dos Sensores usados na EstAcqua}

Na Tabela 1 temos um comparativo entre os sensores conectados à EstAcqua com alguns produtos comerciais da empresa Onset, sendo eles: (i) TidbiT v2 Water Temperature Data Logger $^{10}$; (ii) HOBO U23 Pro v2 External Temperature/Relative Humidity Data Logger ${ }^{11}$; e (iii) HOBO Pendant Temperature/Light 64K Data Logger ${ }^{12}$.

Como mostrado na Tabela 1, podemos verificar que os sensores utilizados na EstAcqua custam aproximadamente $1 \%$ do valor dos produtos comerciais, por isso o prejuízo em caso de furto ou dano dos sensores é extremamente baixo. Além disso, permite a aquisição de novos sensores que forem lançados, não ficando restrito a um fabricante.

Ainda apresentam uma acurácia e resolução próximas dos sensores comerciais e em alguns casos, melhor. Analisando o fundo de escala e temperatura de operação vemos que os sensores da EstAcqua são, na maioria, melhores que os comerciais. Apesar

\footnotetext{
${ }^{10} \mathrm{http}: / / \mathrm{http}: / / \mathrm{www}$. onsetcomp.com/products/data-loggers/utbi-001

${ }^{11} \mathrm{http}: / / \mathrm{http}: / / \mathrm{www}$. onsetcomp.com/products/data-loggers/u23-002

${ }^{12} \mathrm{http}: / / \mathrm{http}: / /$ www.onsetcomp.com/products/data-loggers/ua-002-64
} 
Tabela 1. Comparativo com sensores comerciais

\begin{tabular}{|c|c|c|c|c|c|c|}
\hline & \multicolumn{2}{|c|}{ Luminosidade } & \multicolumn{2}{|c|}{ Temperatura externa e umidade relativa } & \multicolumn{2}{|c|}{ Temperatura submersa } \\
\hline & BH1750 (EstAcqua) & Onset HOBO Pendant & BME280 (EstAcqua) & Onset HOBO U23 Pro & DS18B20 (EstAcqua) & Tidbit v2 \\
\hline Acurácia & $11 \mathrm{x}$ & $\begin{array}{l}\text { Desenvolvido para } \\
\text { medidas relativas }\end{array}$ & $\begin{array}{c}\text { Temperatura: } \pm 1^{\circ} \mathrm{C} \\
\text { Unidade: } \pm 3 \% \\
\end{array}$ & $\begin{array}{c}\text { Temperatura: } \pm 0,21^{\circ} \mathrm{C} \\
\text { Umidade: } \pm 2,5 \% \\
\end{array}$ & $\pm 0,5^{\circ} \mathrm{C}$ & $\pm 0,021^{\circ} \mathrm{C}$ \\
\hline Resolução & $11 \mathrm{x}$ & $11 \mathrm{x}$ & $\begin{array}{l}\text { Temperatura: } 0,0,1^{\circ} \mathrm{C} \\
\text { Umidade: } 0,008 \%\end{array}$ & $\begin{array}{l}\text { Temperatura: } 0,00^{\circ} \mathrm{C} \\
\text { Unidade: } 0,05 \%\end{array}$ & 9 a 12 bits & $0,02^{\circ} \mathrm{C}$ \\
\hline $\begin{array}{l}\text { Temperatura } \\
\text { de operaçăo }\end{array}$ & -40 a $85^{\circ} \mathrm{C}$ & -20 a $70^{\circ} \mathrm{C}$ & -40 a $85^{\circ} \mathrm{C}$ & -40 a $70^{\circ} \mathrm{C}$ & -55 a $125^{\circ} \mathrm{C}$ & -20 a $70^{\circ} \mathrm{C}$ \\
\hline $\begin{array}{l}\text { Fundo de } \\
\text { escala }\end{array}$ & 0 a $65.535 \mathrm{~lx}$ & 0 a $320.000 \mathrm{~lx}$ & $\begin{array}{c}\text { Temperatura: }-40 \text { a } 85^{\circ} \mathrm{C} \\
\text { Umidade: } 0 \text { a } 100 \%\end{array}$ & $\begin{array}{c}\text { Temperatura: }-40 \mathrm{a} 70^{\circ} \mathrm{C} \\
\text { Umidade: } 0 \text { a } 100 \%\end{array}$ & -55 a $125^{\circ} \mathrm{C}$ & -20 a $70^{\circ} \mathrm{C}$ \\
\hline Preço & u\$ 3,15 & Us 64,00 & u\$7,56 & U\$ 199,00 & US 1,37 & US 133,00 \\
\hline Bateria & Recarregável & $\begin{array}{l}\text { Não recarregável, com } \\
\text { duração de } 1 \text { ano }\end{array}$ & Recarregável & $\begin{array}{l}\text { Não recarregável, com } \\
\text { duração de } 3 \text { anos }\end{array}$ & Recarregável & $\begin{array}{l}\text { Não recarregável, com } \\
\text { duração de } 5 \text { anos }\end{array}$ \\
\hline Data Logger & $\operatorname{sim}$ & $\begin{array}{l}\text { Sim. Sobrescreve os } \\
\text { dados se năo forem } \\
\text { coletados em } 30 \text { dias. }\end{array}$ & $\operatorname{sim}$ & $\begin{array}{c}\text { Sim. Sobrescreve os } \\
\text { dados se năo forem } \\
\text { coletados em } 30 \text { dias. }\end{array}$ & sim & $\begin{array}{l}\text { Sim. Sobrescreve os } \\
\text { dados se nåo forem } \\
\text { coletados em } 45 \text { dias. }\end{array}$ \\
\hline $\begin{array}{l}\text { Transmissão } \\
\text { sem fio }\end{array}$ & sim & Não & sim & Não & sim & Não \\
\hline
\end{tabular}

da acurácia e resolução serem em alguns casos, inferiores aos produtos comerciais analisados, não torna a substituição pela EstAcqua inviável, pois para medições ambientais os valores analisados com precisão de uma casa decimal são suficientes conforme, Figura 6, que mostra o gráfico de temperatura gerado pelo equipamento comercial Sontek CastAway-CTD ${ }^{13}$, cujo incremento de temperatura é de $1^{\circ} \mathrm{C}$.

Apesar de possuir autonomia de bateria de aprox. 171 dias, é possível acoplar painel solar portátil em cada nó EstAcqua resultando em autonomia limitada somente pela vida útil de alguns anos da bateria e dos outros componentes eletrônicos.

Por fim, a EstAcqua ainda registra os dados coletados em cartão micro SD e no IoT Cloud, tornando possível a visualização dos dados em tempo real e, em caso de perda ou furto do equipamento, os dados coletados não são perdidos pois já se encontram na IoT Cloud. Vale ressaltar que nas soluções comerciais apresentadas, caso não haja uma coleta regular, os dados começam a ser sobrescritos.

\section{Conclusão}

Podemos concluir pelos resultados obtidos na avaliação dos sensores que a EstAcqua possui sensores de acurácia e resolução bem próximas de sensores comerciais e custam significativamente menos que as soluções encontradas no mercado atualmente. Além disso, a EstAcqua tem como grande vantagem a capacidade de coleta e transmissão dos dados para um NanoGateway situado a quilômetros de distância, podendo acessar os dados via web ou celular, conforme mostra seção de testes de interface de monitoramento via nuvem, cobrindo assim uma lacuna existente nas soluções encontradas atualmente, que além de não possuírem integração com outros dispositivos, são comercializados com baterias não recarregáveis e/ou substituíveis. A EstAcqua ainda conta com uma redundância de armazenamento para os dados, pois mesmo se o sensor falhar, for danificado e/ou furtado, os dados coletados já foram transferidos para a nuvem, diferente das soluções atuais onde se perderiam todos os dados coletados. Os resultados obtidos na avaliação no lago Terra Alta mostram que EstAcqua é uma solução excelente não somente para o uso acadêmico, mas também como um substituto de menor custo, confiável, com maior integração e mais funcionalidades do que a maioria das soluções encontradas no mercado.

Como trabalhos futuros, podemos citar o uso de uma caixa certificada IP67 para que se tenha maior segurança para os nós que ficarão em ambiente externo, o uso de placas

\footnotetext{
${ }^{13}$ https://www.sontek.com/productsdetail.php?CastAway-CTD-11
} 
solares para recarregar a bateria durante o dia fazendo com que a autonomia da bateria seja praticamente a sua vida útil, a adição de mais sensores visando monitorar mais parâmetros ambientais, o uso de downlink para mudar parâmetros da EstAcqua através do IoT Cloud e a realização de novos comparativos com os equipamentos comerciais utilizados pelo departamento de Oceanografia e Ecologia da UFES.

\section{Agradecimentos}

Este trabalho recebeu financiamento parcial do projeto FUTEBOL - Horizon 2020 (União Europeia) sob no. 688941, assim como do MCTI por meio da RNP e do CTIC. Além disso, também gostaríamos de agradecer o financiamento parcial do CNPq e FAPES.

\section{Referências}

Adrian, R., O’Reilly, C. M., Zagarese, H., Baines, S. B., Hessen, D. O., Keller, W., Livingstone, D. M., Sommaruga, R., Straile, D., Donke, E. V., Weyhenmeyer, G. A., and Winder, M. (2009). Lakes as sentinels of climate change. Limnology and Oceanography, 54:2283-2297.

Barroso, G. F., d. C. Garcia, F., Gonçalves, M. A., Martins, F. C. O., Venturini, J. C., d. C. Sabadini, S., d. Azevedo, A. K., d. Freitas, A. C. T., and Delazari-Barroso, A. (2012). Estudos integrados no sistema lacustre do Baixo Rio Doce (Espírito Santo). I Seminário Nacional de Gestão Sustentável de Ecossistemas Aquáticos: Complexidade, Interatividade e Ecodesenvolvimento.

Bharathkumar, V., Irshad, S., Gowtham, S., and Geethamani, R. (2017). Microcontroller based digital meter with alert system using GSM. 2017 11th International Conference on Intelligent Systems and Control (ISCO), pages 444-448.

de Oliveira, F. F. (2017). EstGeoMag: Integrando soluções de Hardware, Software e Internet das Coisas na medição de grandezas Geomagnéticas. Sociedade Brasileira de Computação, pages 2598-2609.

Loureiro, A. A. F. (2016). Minicursos XXXIV Simpósio Brasileiro de Redes de Computadores e Sistemas Distribuídos. In Allan Edgard Silva Freitas, Fabíola Gonçalves Pereira Greve, F. A. S. and Lung, L. C., editors, Simpósio Brasileiro de Redes de Computadores e Sistemas Distribuídos, pages 1-50. Sociedade Brasileira de Computação.

Mois, G., Folea, S., and Sanislav, T. (2017). Analysis of three IoT-Based wireless sensors for environmental monitoring. IEEE Transactions on Instrumentation and Measurement, 66(8):2056-2064.

Schindler, D. W. (2009). Lakes as sentinels and integrators for the effects of climate change on watersheds, airsheds and landscapes. Limnology and Oceanography, 54:2349-2358.

Straskraba, M. and Tundisi, J. G. (2000). Gerenciamento da qualidade da água em reservatórios. International Lake Environmental Committee, page 280.

Tose, T. (2012). Rede de Sensores sem fio Zigbee Aplicada em uma Estação de Tratamento de Esgoto. Master's thesis, Universidade Federal do Espírito Santo, Av. Fernando Ferrari, 514 - Goiabeiras, Vitória - ES, 29075-073. 